\title{
Echocardiographic evaluation of total anomalous pulmonary venous connection: comparison of obstructed and unobstructed type
}

\section{Yonghua Xiang}

Hunan Children's Hospital

\section{Xuehua Zhang}

Hunan Children's Hospital

Jun Qiu

Hunan Children's Hospital

Qing Gan

Hunan Children's Hospital

Ke Jin ( $\nabla$ jkkj2009@163.com )

Hunan Children's Hospital https://orcid.org/0000-0002-9342-670X

Research article

Keywords: Total anomalous pulmonary venous connection; echocardiography; pulmonary venous obstruction; comparison

Posted Date: July 25th, 2019

DOI: https://doi.org/10.21203/rs.2.11895/v1

License: (c) (i) This work is licensed under a Creative Commons Attribution 4.0 International License. Read Full License 


\section{Abstract}

Purpose The purpose of this study was to compare the differences between obstructed and unobstructed TAPVC using echocardiography, then to predict PVO by assessing clinical and echocardiographic parameters. Methods We conducted a retrospective study included 70 patients with TAPVC between 2014 and 2018. The morphological and hemodynamic echocardiographic parameters were observed and measured. The clinical and echocardiographic parameters that existed difference between obstructed and unobstructed TAPVC were selected to predict PVO by running ROC curve analysis. Results Between obstructed and unobstructed TAPVC, there were significant differences in ASD size, PA Vmax, MV VE, LVFS, LVEF, SV and the incidence of PDA, but there was no significant difference in birth weight. The first admission age of obstructed TAPVC was earlier than unobstructed type. The ROC curve analysis for the first admission age in predicting PVO showed the sensitivity and specificity were $76.7 \%, 80 \%$ respectively. The ROC curve analysis for multiple echocardiographic parameters showed the sensitivity and specificity were $82.6 \%, 100 \%$ respectively. Conclusions The presence of PVO led to anatomical and functional abnormity of patients with TAPVC, and then led to hospitalize earlier. The first admission age was a simple and feasible parameter in predicting PVO. The integrated use of multiple echocardiographic parameters had an excellent value in predicting PVO.

\section{Introduction}

TAPVC is an uncommon malformation of congenital heart disease in which all pulmonary veins connect directly to the systemic venous circulation, and not the $\mathrm{LA}^{[1]}$. Depending on the drainage site of the pulmonary veins, the defect can be divided into four anatomic types: supracardiac, cardiac, infracardiac and mixed type. Pathophysiologically, these four types are subclassified into obstructed and unobstructed TAPVC according to whether there is a PVO in drainage pathway. PVO can alter hemodynamics of patients with TAPVC, and then to affect the clinical presentation, physiology and prognosis, so whether existing a PVO is critical for the timing of surgery and surgical management ${ }^{[2,3]}$. Echocardiography is regarded as the preferred initial screening and diagnostic modality in patients with TAPVC, but there are few comparative studies of echocardiographic parameters between obstructed and unobstructed TAPVC at present. In addition, PVO may occur at any point along the anomalous pathway, including the VV, the portal venous system, the ductus venous, the level of the hepatic sinusoids and so on ${ }^{[4]}$, and an incorrect diagnosis by echocardiography might occur because of an insufficient spatial resolution, restricted acoustic window or an interpretation error by the operators ${ }^{[5,6]}$. We have often experienced difficulty in evaluating the PVO outside the mediastinum. The aims of this study were to compare the differences between obstructed and unobstructed TAPVC using echocardiography, to seek the correlations between these observational indicators, then to predict PVO by assessing the clinical and echocardiographic parameters.

\section{Patients And Methods}




\section{Patients}

Patients with TAPVC were consecutively collected in Hunan Children's Hospital from 2014 to 2018. A total of 70 cases were included in the study, 47 males and 23 females, aged from 1 day to 5 years, median age was 23 days. It was classified into obstructed and unobstructed TAPVC according to whether there was a PVO by CTA or surgical results, including 30 obstructed and 40 unobstructed types. PVO (obstructed TAPVC) was considered if the drainage veins were reduced by $50 \%$ or more from the largest measured dimension. Drainage veins connecting to portal vein were also taken as an indicator of PVO, because obstruction results from the high resistance of portal venous flow needing to traverse through the liver parenchyma before returning to the heart ${ }^{[7]}$. Cases with functionally univentricular circulation, common atrium or pulmonary atresia were excluded in predicting PVO. CTA was reviewed by two experienced cardiac radiologist to confirm whether there is a PVO.

\section{Echocardiography}

Echocardiographic examinations were performed with PHILIPS EPIQ 7C ultrasound systems equipped with phased array probe (S8-3), including two-dimensional, pulsed wave and color Doppler examinations. All studies were performed by an experienced cardiac echocardiographer and reviewed by another pediatric cardiologist with more than ten years of experience in cardiac imaging.

Echocardiographic parameters were measured according to the guidelines of the American Society of Echocardiography (ASE). The morphological and hemodynamic echocardiographic parameters were recorded as the observation indicators. For parameters affected by age, the ratios were calculated to minimize the effect of age. We calculated the ratio of diameter of pulmonary artery/ascending aorta (PA/AA), right/left ventricular inner diameter (RD/LD) and right / left ventricular wall thickness (RT/LT). The clinical and echocardiographic parameters that existed significant differences between obstructed and unobstructed TAPVC were selected to predict PVO.

\section{Statistical analysis}

Data were collected and analyzed with SPSS18. Normally distributed continuous variables were described as mean \pm SD. Student $t$ tests were used to compare the differences between groups. Descriptive statistics for categorical variables were reported as frequency/percentage and were compared by using of the Chi-Square test $\left({ }^{2}\right)$. The ROC curve analysis was used to predict PVO. When running the ROC curve analysis for multiple variables (seven echocardiographic parameters) in predicting PVO, the first step was to create a logistic regression model using the seven echocardiographic parameters, and yielded a new variable that contains individual predicted probabilities in the data view, and then the new variable was used to perform the ROC curve analysis as a test variable. Values of $P \otimes 0.05$ were considered statistically significant.

\section{Results}


Obstructed TAPVC was found in $30(42.9 \%)$ of 70 patients in this study. PVO occurred in 22(52.4\%) of 42 supracardiac, $0(0 \%)$ of 13 cardiac, $7(87.5 \%)$ of 8 infracardiac, and $1(14.3 \%)$ of 7 mixed types. There was significant difference of the incidence of PVO among the four anatomic types $\left({ }^{2}=20.149, P \otimes 0.001\right)$. The first admission age of obstructed TAPVC was earlier than the unobstructed type, and patients with PVO were usually hospitalized during neonatal period (table 1). The average birth weights of patients with obstructed and unobstructed TAPVC were 3.27 and $3.13(\mathrm{~kg})$ respectively, and there was no significant difference between the two groups $(t=1.573, P=0.120)$. Eleven echocardiographic parameters were compared between obstructed and unobstructed TAPVC (table 2). There were significant differences between obstructed and unobstructed TAPVC in ASD size, PA $V_{\text {max }}, M V V_{E}$, LVFS, LVEF, SV and whether a PDA. These seven echocardiographic parameters with significant difference were used to predict PVO.

The results of ROC curve analysis to predict PVO by a single variable were summarized in table 3 . The first admission age as one of the easiest indicators to obtain was efficient to predict PVO. The ROC curve analysis for the first admission age (figure 1 ) showed the area under the curve was 0.805 , and the sensitivity and specificity were $76.7 \%, 80 \%$ respectively at the optimal cut-off value of 0.64 (months). The other seven echocardiographic parameters appeared to be limited in its predictive ability in predicting PVO independently. But the integrated use of multiple echocardiographic parameters to predict PVO can greatly improve the efficiency of diagnosis. There was an excellent value in predicting PVO when a logistic model was created using the seven echocardiographic parameters (table 4). The ROC curve analysis for multiple echocardiographic parameters in predicting PVO (figure 2) showed the area under the curve was 0.936 , and the sensitivity and specificity were $82.6 \%, 100 \%$ respectively at the optimal cutoff value of 0.5774 (predicted probability P).

\section{Discussion}

Obstructed TAPVC was found in $25 \%$ to $50 \%$ of patients with TAPVC, and represents a life-threatening neonatal emergency warranting immediate surgery ${ }^{[8-10]}$. The incidence of PVO was highest in the infracardiac and lowest in the cardiac type ${ }^{[11,12]}$. Generally, the longer of the drainage vein connecting to RA, the higher incidence of PVO. The infracardiac and cardiac types have the longest and shortest drainage vein respectively of four types. In this study, PVO was found in $42.9 \%$ of patients, and the PVO occurred in $87.5 \%$ of infracardiac, $52.4 \%$ of supracardiac, but none of cardiac type presents PVO $(0 \%)$. In supracardiac, the most common sites of PVO were at the level of VV, especially at the place where the VV step over the left PA and bronchus, and the $V V$ was often oppressed by them. In infracardiac, the drainage veins were usually connected to $P V$, and obstruction resulted from the high resistance of PV flow needing to traverse through the liver parenchyma before returning to $\operatorname{RA}^{[7,13,14]}$.

Our study showed the patients with TAPVC had normal birth weight, and there was no significant difference in birth weight between obstructed and unobstructed TAPVC. The reason may be the unique nature of fetal hemodynamics allows the fetus with TAPVC to be well tolerated in utero. Pulmonary blood 
flow of the fetus was a small portion of the combined ventricular output, so the physical growth of the fetus will not be affected in utero. However, there was complete mixing of the pulmonary and systemic circulations in the right heart after birth. The patients will be cyanotic and may have difficulty feeding in the first weeks or months of life. If there was a PVO, it resulted in elevated pulmonary pressures and decreased systemic oxygen delivery, and severe respiratory distress and decompensation may ensue very early in the neonatal period. The patient's conditions can deteriorate rapidly and become life threatening unless the cause was promptly recognized and treated ${ }^{[15]}$. Our study demonstrated that the first admission age of obstructed TAPVC was earlier than the unobstructed type, and patients with PVO were usually hospitalized during neonatal period. We believed that the presence of PVO led to earlier symptoms and thus to be discovered earlier.

For patients with TAPVC, a right-to-left intracardiac shunt is obligatory for survival, and it almost always occurs at atrial level through an ASD that is rarely restrictive. ASD size is one of the most important factors affecting the survival period. The presence of a large ASD allows some of the excessive venous return in RA to reach LA. Several cases of adult untreated TAPVC have been reported, even a few cases were diagnosed after 50 years of age, and they have very similar pathophysiology to a large ASD ${ }^{[16-18]}$. All our patients presented ASD of various sizes except a case with common atrium, and unobstructed TAPVC had a larger ASD size than obstructed type. The first admission age of unobstructed TAPVC was later than obstructed type, so a larger ASD size maybe resulted in later admission age. PDA was the common concurrent abnormality with an incidence of $44.3 \%$ in this study. The direction of PDA flow was right to left or Bi-directional shunt in 26 of 31 patients. We found that obstructed TAPVC had a higher incidence of PDA than unobstructed type, and the patients with PDA had a smaller ASD than that without PDA. The presence of PDA may relate to pulmonary pressure. Obstruction of drainage vein caused elevated pressure in pulmonary venous territory, elevated pressure in pulmonary capillary bed, pulmonary edema, pulmonary hypertension, and right heart failure with leftward shift of the interventricular septum and low systemic output. Remaining a PDA can alleviate the pulmonary hypertension and provide more blood flow for systemic circulation.

Pulmonary arterial blood flow velocity was related to right ventricular function, pulmonary arterial pressure, blood flow and arterial diameter and so on. Right ventricular volume overload and pulmonary hypertension were common findings in patients with TAPVC ${ }^{[19]}$, and patients with TAPVC were often found having abnormal pulmonary artery flow velocity. Our study demonstrated that PA Vmax significantly higher in patients with unobstructed TAPVC than obstructed type. Obstructed type had a lower PA Vmax, which maybe resulted from increased pulmonary vascular resistance and decreased compliance due to obstructed drainage veins. However, unobstructed type had an accelerated PA Vmax, we speculated that the increase of pulmonary circulation blood flow was not compatible with the expanding pulmonary artery itself, resulting in functional pulmonary artery stenosis, then led to an accelerated PA Vmax.

Enlargement of the right heart was a common feature to patients with TAPVC. The RV was disproportionately larger than the LV in most of our cases. The LA was frequently small and the LV was 
compressed by the dilated RV. The ratios of RD/LD of obstructed and unobstructed TAPVC were larger than normal, but there was no significant difference between obstructed and unobstructed type. For patients with TAPVC, the blood of left heart was almost always obtained by an ASD, so the size of ASD could often affect the LV volume. The presence of PVO reduced the blood flow returning into the RA, the obligatory right-to-left shunt was also attenuated and the LV volume became small. The obstructed TAPVC had a smaller ASD than unobstructed type, which resulted in decreasing of SV in patients with obstructed TAPVC. Our study demonstrated that the obstructed TAPVC had a higher LVFS and LVEF than unobstructed type. The main reason may be that obstructed TAPVC need to increase cardiac output through strengthening the ventricular systole.

TAPVC has an excellent outcome if detected early and corrected surgically in due time, but the prognosis is poor when TAPVC is associated with a PVO ${ }^{[20]}$. The presence of PVO was significantly associated with an increased risk of death ${ }^{[3,8]}$. Therefore, an important part of preoperative diagnosis of TAPVC was to determine whether there was a PVO. Echocardiography can detect the PVO by measuring the blood velocity of the stenotic pulmonary vein ${ }^{[21]}$. But incorrect diagnosis might occur because of an insufficient spatial resolution, restricted acoustic window or an interpretation error by the operators. The role of echocardiography was limited in the evaluation of obstruction, especially in patients with infracardiac and mixed TAPVC ${ }^{[19]}$. We tried to predict PVO by some familiar, easily obtained echocardiographic and clinical parameters. The ROC curve analysis was used to predict PVO in this study. To the best our knowledge, it was the first time to determine PVO using this method. The first admission age of obstructed TAPVC was earlier than the unobstructed type. The ROC curve analysis for the first admission age showed the area under the curve was 0.805 , and the sensitivity and specificity were $76.7 \%, 80 \%$ respectively at the optimal cut-off value of 0.64 (months). Therefore, we can make a preliminary judgment whether there was a PVO according to the first admission age, which was the easiest parameter to obtain. If the first admission age was less than 0.64 months, then a suspicion of PVO was strongly suggested, and further imaging examination was necessary. For both clinicians and echocardiographer, this was a convenient and quick way to understand the patient's conditions. Among echocardiographic parameters, the PA Vmax had $88.5 \%$ sensitivity and $67.6 \%$ specificity at the optimal cut-off value of $1.11(\mathrm{~m} / \mathrm{s})$, and the area under the curve was 0.841 . The other separate parameters appeared to be limited in its predictive ability. Compared to a single parameter, the comprehensive utilization of multiple echocardiographic parameters can greatly improve the efficiency of diagnosis. There was an excellent value in predicting PVO when a logistic model was created using the seven echocardiographic parameters. The area under the curve was 0.936 , and the sensitivity and specificity were $82.6 \%, 100 \%$ respectively at the optimal cut-off value of 0.5774 . This was a simple method to predict PVO, and it required measurements of only some familiar and easily obtained data.

\section{Conclusions}

Patients with TAPVC had a high incidence of PVO, especially in the infracardiac type, whereas cardiac type was less likely to occur. The presence of PVO led to anatomical and functional abnormity of patients 
with TAPVC, and then led to hospitalize earlier. The first admission age was a simple and feasible parameter in predicting PVO, and we can use it to make a preliminary judgment. The integrated use of multiple echocardiographic parameters had an excellent value in predicting PVO.

\section{Abbreviations}

TAPVC = total anomalous pulmonary venous connection, $\mathrm{PVO}=$ pulmonary venous obstruction, $\mathrm{ROC}=$ receiver operating characteristic, $\mathrm{ASD}=$ atrial septal defect, $\mathrm{PA}=$ pulmonary artery, $\mathrm{PA} \mathrm{V}_{\max }=$ pulmonary artery maximum velocity, $A A V_{\max }=$ ascending aorta maximum velocity, $M V V_{E}=$ Peak $E$ velocity of mitral valve, LVFS = left ventricular fractional shortening, LVEF = left ventricular ejection fraction, SV = stroke volume, PDA = patent ductus arteriosus, VV = vertical vein, CTA = computed tomography angiography, $\mathrm{PA} / \mathrm{AA}=$ the ratio of diameter of pulmonary artery/ascending aorta, $\mathrm{RD} / \mathrm{LD}=$ the ratio of right /left ventricular inner diameter, RT/LT = the ratio of right/left ventricular wall thickness. $\mathrm{RA}=$ right atrium, $\mathrm{LA}=$ left atrium, $P V=$ portal vein, $R V=$ right ventricle, $L V=$ left ventricle.

\section{Declarations}

Ethics approval and consent to participateखThis study was approved by the Ethics Committee of Hunan Children's Hospital with a waiver of informed consent.

Consent for publicationखNot applicable.

Availability of data and material: The datasets used and/or analysed during the current study are available from the corresponding author on reasonable request.

Competing interests: The authors declare that they have no competing interests.

Funding: This study was supported by Health and Family Planning Commission of Hunan Province (C2019023).

Authors' contributions: All authors read and approved the final manuscript.

Acknowledgements: Not applicable.

\section{References}

1. Byard RW, Gilbert JD (2005) Total anomalous pulmonary venous connection: Autopsy considerations. Forensic Sci Med Pathol 1:215-220

2. Hoashi T, Kagisaki K, Kurosaki K et al (2015) Intrinsic obstruction in pulmonary venous drainage pathway is associated with poor surgical outcomes in patients with total anomalous pulmonary venous connection. Pediatr Cardiol 36:432-437 
3. Shi GC, Zhu ZQ, Chen JM et al (2017) Total anomalous pulmonary venous connection: the current management strategies in a pediatric cohort of 768 patients. Circulation135:48-58

4. Ho ML, Bhalla S, Bierhals A et al (2009) MDCT of partial anomalous venous return (PAPVR) in adults. $\mathrm{J}$ Thorac Imaging 24:89-95

5. Zhang Z, Zhang L, Xie F et al (2016) Echocardiographic diagnosis of anomalous pulmonary venous connections: Experience of 84 cases from 1 medical center. Medicine 95:e5389

6. Oh KH, Choo KS, Lim SJ et al (2009) Multidetector CT evaluation of total anomalous pulmonary venous connection: comparison with echocardiography. Pediatr Radiol 39:950-954

7. Stein P (2009) Total anomalous pulmonary venous connection. AORN J 85:509-520

8. Karamlou T, Gurofsky R, Al Sukhni EA et al (2007) Factors associated with mortality and reoperation in 377 children with total anomalous pulmonary venous connection. Circulation 115:1591-1598

9. Husain SA, Maldonado E, Rasch D et al (2012) Total anomalous pulmonary venous connection: factors associated with mortality and recurrent pulmonary venous obstruction. Ann Thorac Surg 94: 825-831

10. Horer J, Neuray C, Vogt M et al (2013) What to expect after repair of total anomalous pulmonary venous connection: data from 193 patients and 2902 patient years. Eur J Cardiothorac Surg 44: 800-807

11. Dillman JR, Yarram SG, Hernandez RJ (2009) Imaging of pulmonary venous developmental anomalies. AJR Am J Roentgenol 192:1272-1285

12. Seale AN, Uemura $H$, Webber $S A$ et al (2010) Total anomalous pulmonary venous connection: morphology and outcome from an international population-based study. Circulation 122:2718-2726

13. Goo HW, Park IS, Ko JK et al (2003) CT of congenital heart disease: normal anatomy and typical pathologic conditions. Radiographics 23:S147-S165

14. Livolsi A, Kastler B, Marcellin L et al (1991) MR diagnosis of subdiaphragmatic anomalous pulmonary venous drainage in a newborn. $J$ Comput Assist Tomogr 15:1051-1053

15. Hancock Friesen CL, Zurakowski D, Thiagarajan RR et al (2005) Total anomalous pulmonary venous connection: an analysis of current management strategies in a single institution. Ann Thorac Surg 79:596-606

16. Ogawa M, Nakagawa M, Hara M et al (2013) Total anomalous pulmonary venous connection in a 64year-old man: a case report. Ann Thorac Cardiovasc Surg 19:46-48

17. McMullan MH, Fyke FE (1992) Total anomalous pulmonary venous connection: surgical correction in a 66-year-old man. Ann Thorac Surg 53:520-521

18. Feng Q, Wu S, Yu G (2010) Surgical treatment of a 56-year-old woman with an intracardiac type of total anomalous pulmonary venous connection. Thorac Cardiovasc Surg 58:175-176

19. Shen Q, Pa M, Hu X et al (2013) Role of plain radiography and CT angiography in the evaluation of obstructed total anomalous pulmonary venous connection. Pediatr Radiol 43:827-835 
20. Padalino MA, Cavalli G, De Franceschi M et al (2014) Surgical outcomes of total anomalous pulmonary venous connection repair: a 22-year experience. J Card Surg 29: 678-685

21. Ohtsuki S, Baba K, Kataoka K et al (2005) Usefulness of helical computed tomography in diagnosing pulmonary vein stenosis in infants. Acta Med Okayama 59:93-98

\section{Tables}

Table 1. Comparison of the first admission age between obstructed and unobstructed TAPVC.

\begin{tabular}{|c|c|c|c|c|c|}
\hline Groups & $\mathrm{N}$ & Admission age & & $\begin{array}{l}\chi^{2} \\
\square P \text { value } \mathbb{}\end{array}$ & OR \\
\hline & & $\begin{array}{l}\text { Neonatal } \\
\text { ( } \leq 28 \text { days) }\end{array}$ & $\begin{array}{l}\text { Non neonatal } \\
\text { ( } ₫ 28 \text { days) }\end{array}$ & & \\
\hline Obstructed & 30 & $24 \llbracket 80.0 \% \rrbracket$ & $6 \rrbracket 20.0 \% \rrbracket$ & $15.52 \otimes 0.000 \rrbracket$ & 2.46 \\
\hline Unobstructed & 40 & $13 \varangle 32.5 \% \rrbracket$ & $27 \rrbracket 67.5 \% \bigotimes$ & & \\
\hline Total & 70 & 37 & 33 & & \\
\hline
\end{tabular}

OR, odds ratio.

Table 2. Comparison of the echocardiographic parameters between obstructed and unobstructed TAPVC. 


\begin{tabular}{|c|c|c|c|c|c|}
\hline Variables & $\mathrm{N}$ & PVO (mean \pm SD) & & $\begin{array}{l}\text { T test } \\
\text { (or 2) }\end{array}$ & $P$ value \\
\hline & & obstructed & unobstructed & & \\
\hline $\mathrm{PA} / \mathrm{AA}$ & 69 & $1.70 \pm 0.24$ & $1.57 \pm 0.29$ & 1.979 & 0.052 \\
\hline $\mathrm{RD} / \mathrm{LD}$ & 67 & $1.55 \pm 0.36$ & $1.63 \pm 0.36$ & -0.947 & 0.347 \\
\hline RT/LT & 69 & $1.55 \pm 0.45$ & $1.41 \pm 0.34$ & 1.402 & 0.167 \\
\hline ASD $(\mathrm{mm})$ & 69 & $7.21 \pm 1.91$ & $9.98 \pm 4.37$ & -3.192 & 0.002 \\
\hline PA Vmax (m/s) & 63 & $0.89 \pm 0.32$ & $1.68 \pm 1.08$ & -3.597 & 0.001 \\
\hline$A A \operatorname{Vmax}(m / s)$ & 63 & $0.87 \pm 0.19$ & $0.96 \pm 0.18$ & -1.941 & 0.057 \\
\hline $\mathrm{MV} V E \rrbracket \mathrm{m} / \mathrm{s} \rrbracket$ & 63 & $0.92 \pm 0.16$ & $1.01 \pm 0.13$ & -2.366 & 0.022 \\
\hline LVFS (\%) & 63 & $42.3 \pm 10.0$ & $36.1 \pm 7.1$ & 2.749 & 0.008 \\
\hline LVEF (\%) & 63 & $76.1 \pm 10.1$ & $68.8 \pm 9.2$ & 2.85 & 0.006 \\
\hline SV $(\mathrm{ml})$ & 63 & $2.97 \pm 1.63$ & $5.67 \pm 3.96$ & -3.147 & 0.003 \\
\hline Exist a PDA $(n, \%)$ & 70 & $21 \otimes 70 \% \bigotimes$ & $10 \rrbracket 25 \% \rrbracket$ & $14.07 \rrbracket$ & 0.000 \\
\hline
\end{tabular}

叉,Chi-Square test.

Table 3. The ROC curve analysis in predicting PVO by a single predictor. 


\begin{tabular}{|c|c|c|c|c|c|c|}
\hline Predictors & $\begin{array}{l}\text { Cut-off } \\
\text { points }\end{array}$ & $\begin{array}{l}\text { Sensitivity } \\
\square \% \bigotimes\end{array}$ & $\begin{array}{l}\text { Specificity } \\
\square \% \bigotimes\end{array}$ & $\begin{array}{l}\text { Areas } \\
\text { un- } \\
\text { der } \\
\text { curve }\end{array}$ & $95 \% \mathrm{Cl}$ & $\begin{array}{l}P \\
\text { value }\end{array}$ \\
\hline $\mathrm{ASD} \otimes \mathrm{mm} \rrbracket$ & $8.85^{\star}$ & 82.8 & 55.0 & 0.707 & $\begin{array}{l}0.584- \\
0.830\end{array}$ & 0.003 \\
\hline PDA (exist=1) & 1 & 70.0 & 75.0 & 0.725 & $\begin{array}{l}0.602- \\
0.848\end{array}$ & 0.001 \\
\hline $\mathrm{PA} V \mathrm{max} \otimes \mathrm{m} / \mathrm{s} \rrbracket$ & $1.11 *$ & 88.5 & 67.6 & 0.841 & $\begin{array}{l}0.744- \\
0.939\end{array}$ & 0.000 \\
\hline LVFS『\%ه & $40.5 \#$ & 54.2 & 78.8 & 0.658 & $\begin{array}{l}0.509- \\
0.808\end{array}$ & 0.043 \\
\hline LVEF®\%囚 & $75.5 \#$ & 54.2 & 78.8 & 0.668 & $\begin{array}{l}0.520- \\
0.816\end{array}$ & 0.032 \\
\hline $\mathrm{SV}(\mathrm{ml})$ & $3.1^{\star}$ & 70.8 & 75.8 & 0.751 & $\begin{array}{l}0.622- \\
0.879\end{array}$ & 0.001 \\
\hline MV VE\m/s $\rrbracket$ & $0.925^{\star}$ & 51.9 & 82.9 & 0.671 & $\begin{array}{l}0.532- \\
0.831\end{array}$ & 0.021 \\
\hline $\begin{array}{l}\text { Admission age } \\
\text { (Mon) }\end{array}$ & $0.64 *$ & 76.7 & 80.0 & 0.805 & $\begin{array}{l}0.698- \\
0.912\end{array}$ & 0.000 \\
\hline
\end{tabular}

$\mathrm{Cl}$, confidence interval; * Smaller parameters represent more definitive test; \# Larger parameters represent more definitive test; Mon, months.

Table 4. The logistic regression model using multiple echocardiographic parameters to predict PVO was created.

\begin{tabular}{|c|c|c|c|c|c|c|}
\hline & Predictors & B & S.E. & $P$ value & $\operatorname{Exp}(B)$ & $95 \% \mathrm{Cl}$ \\
\hline $\mathrm{X} 1$ & ASD $₫ \mathrm{~mm} \rrbracket$ & -0.284 & 0.187 & 0.129 & 0.752 & $0.521-1.086$ \\
\hline $\mathrm{X} 2$ & PDA (exist=1) & 0.921 & 1.067 & 0.388 & 2.512 & $0.311-20.315$ \\
\hline $\mathrm{x} 3$ & $\mathrm{PA} V \max \otimes \mathrm{m} / \mathrm{s} \rrbracket$ & -3.960 & 1.756 & 0.024 & 0.019 & $0.001-0.595$ \\
\hline $\mathrm{X} 4$ & LVFS『\%囚 & 0.009 & 0.418 & 0.983 & 1.009 & $0.444-2.292$ \\
\hline$x 5$ & LVEF®\%】 & 0.142 & 0.319 & 0.657 & 1.152 & $0.616-2.155$ \\
\hline$x 6$ & $\mathrm{SV}(\mathrm{ml})$ & -0.158 & 0.195 & 0.419 & 0.854 & $0.583-1.252$ \\
\hline $\mathrm{X7}$ & 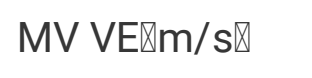 & -3.357 & 4.340 & 0.439 & 0.035 & $0.000-72.217$ \\
\hline & Constant & -0.465 & 8.528 & 0.957 & 0.628 & \\
\hline
\end{tabular}


Logistic regression equation can be obtained from the table: ( $P=$ predicted probability)

$\mathrm{P}=1 /[1+\mathrm{e}-(-0.456-0.284 \times 1+0.921 \times 2-3.960 \times 3+0.009 \times 4+0.142 \times 5-0.158 \times 6-3.357 \times 7)]$.

\section{Figures}

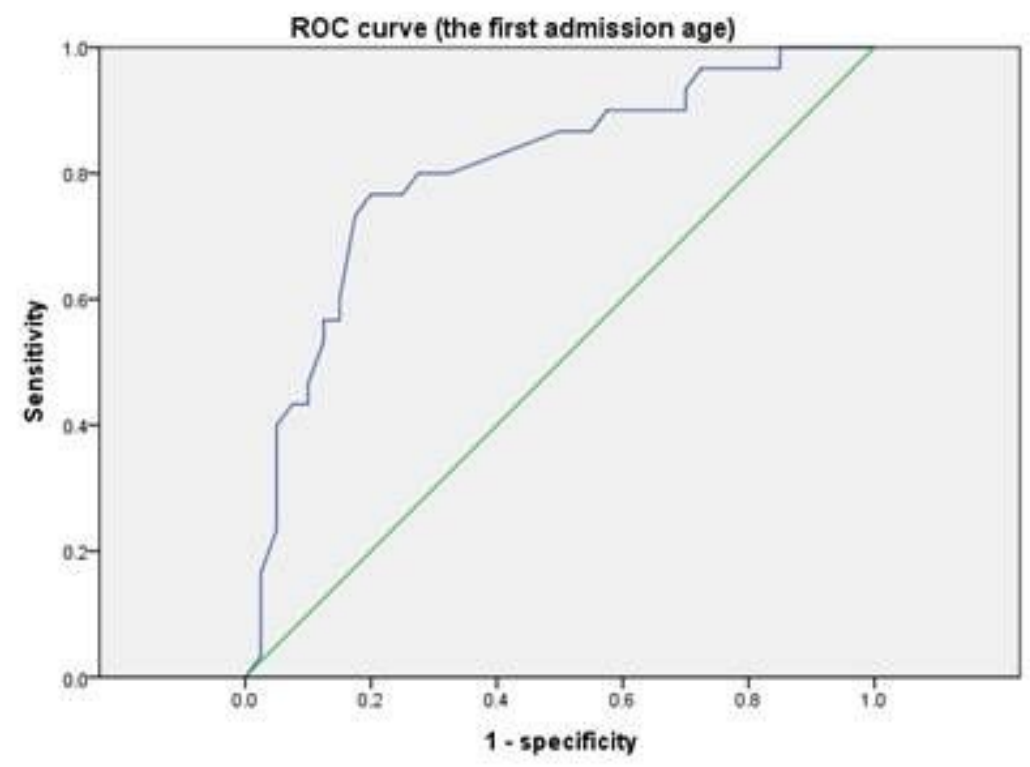

\section{Figure 1}

The ROC curve analysis for the first admission age (month) in predicting PVO; the area under the curve was 0.805 ; the sensitivity was $76.7 \%$ and the specificity was $80 \%$ at the optimal cut-off value of 0.64 (months). Smaller parameters represent more definitive test.

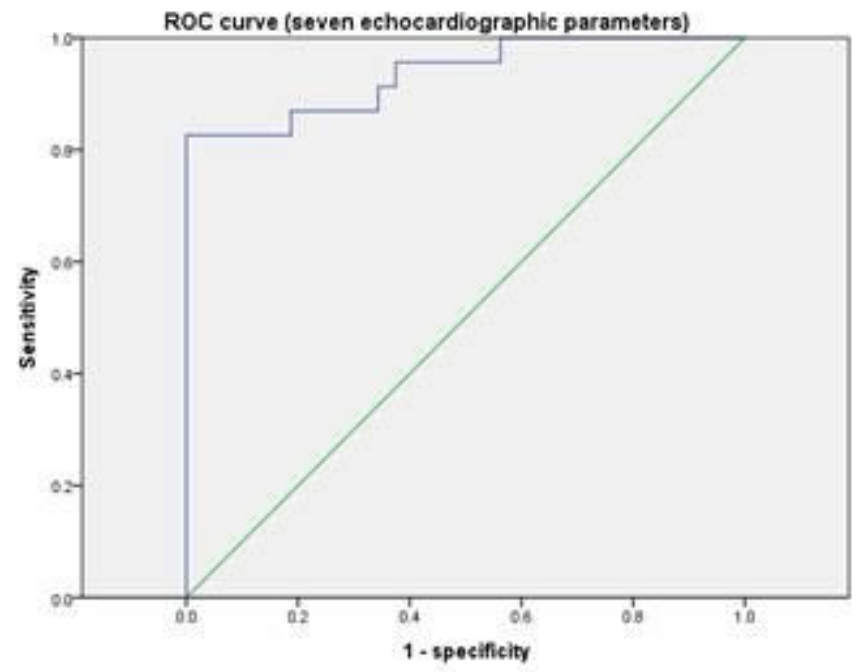

Figure 2 
The ROC curve analysis for multiple echocardiographic parameters in predicting PVO; Area under the curve $=0.936$; Standard error $=0.034 ; \mathrm{P} \otimes 0.001 ; 95 \% \mathrm{Cl}: 0.865-1.000$; the sensitivity was $82.6 \%$ and the specificity was $100 \%$ at the optimal cut-off value of $0.5774(\mathrm{P})$; Larger parameters represent more definitive test. 